\title{
Automatic classification of circuit topologies of appliances based on higher order statistic
}

\author{
Olivia Florencias-Oliveros ${ }^{1}$, Ana María Blanco ${ }^{2}$, Jan Meyer ${ }^{2}$, Juan-José González-de-la-Rosa ${ }^{1}$ and \\ Agustín Agüera-Pérez ${ }^{1}$ \\ ${ }^{1}$ Research Group PAIDI-TIC-168: Computational Instrumentation and Industrial Electronics. \\ Higher Polytechnic School of Algeciras. University of Cadiz, Spain \\ olivia.florencias@uca.es,juanjose.delarosa@uca.es, agustin.aguera@uca.es \\ 2 Institute for Electrical Power Systems and High Voltage Engineering \\ Technische Universität Dresden, Germany \\ ana.blanco@tu-dresden.de,jan.meyer@tu-dresden.de
}

\begin{abstract}
Electronic devices have a non-linear characteristic and are sources of harmonic emission. Their massive use pollutes the network and consequently it is needed to measure and characterize those devices. Harmonic current emitted by electronic devices is closely linked to their circuit topology and the distortion of the supply voltage. Different circuit topologies have also different current waveforms. This paper proposes an automatic classification method of steady-state appliances based on current waveform characterization in the higherorder statistics space. The translation from the time domain to a statistical space enables the automatic identification of individual devices. The algorithm has been applied to the current waveforms of a large set of household appliances measured under sinusoidal conditions. The classification analysis proves that clusters of circuit topologies can be clearly identified. In addition, authors show that kurtosis and variance of an individual cycle provide enough information about the distribution of a waveform shape with respect to its average value, while the skewness inform about the half cycle bias. The method can be a useful tool to identify prevailing circuit topologies in the market. It can also improve automatic load identification, e.g. part of the future intelligent measurement systems such as smart meters.
\end{abstract}

\section{Key words}

Power quality (PQ); harmonic emission, appliance identification; higher-order statistics (HOS); electronic appliances, smart metering

\section{Introduction}

Energy saving programs have promoted the development and usage of electronic devices. Electronic devices are non-linear devices that inject harmonic currents into the network, which may cause undesirable effects on other system elements or users. The impact of an individual electronic device in the network can be negligible. Nevertheless, the effect of thousands of such devices is considered an important challenge and has to be carefully analysed by harmonic studies.

Electronic appliances have different circuit topologies and consequently different current waveforms and different harmonic emission [1], [2]. In order to analyse the effect of multitude of electronic devices in the network, it is indispensable to classify first the multitude of electronic appliances in certain number of categories, and develop models according to each equipment category. The models of equipment categories will simplify and facilitate the harmonic studies.

This paper proposes a method to classify the current waveforms of different household appliances based on time domain features. The main goal is to apply higherorder statistic indices to classify electronic appliances with stable operation measured under reference conditions (measurements under sinusoidal supply voltage and normal operating conditions).

In this sense, higher-order statistics (HOS) can help in the classification, as they are tools capable to depict the shape of a waveform. HOS have been used before in power quality studies in order to characterise symmetry and shape of the voltage waveform distributions (bimodal distributions) via the $3^{\text {rd }}$ and $4^{\text {th }}$ order cumulates [3]. HOS also account for the nonlinear assessment of the signal [4] and are less sensitive to noise, which is good for nonlinear system characterization [5] [6].

The paper is divided in three main parts. The first part introduces the typical circuit topologies of household electronic appliances and gives the theoretical background of HOS. The second part describes the measurements used for the analysis, and gives a detail explanation of the proposed HOS indices in the time domain. The third part presents the analysis results with $2 \mathrm{D}$ diagrams for the 
different analysis scenarios under sinusoidal conditions. Finally, some conclusions and recommendations related to the application of the method are proposed.

\section{Theoretical background}

\section{A. Circuit topologies}

Electronic household appliances have different circuit topologies, which results in different current waveforms (i.e. current distortions), as shown exemplary in fig. 1a-1c for four different appliances. The electronic household appliances have been classified previously based on the type of power factor correction (PFC) implemented in the device:

No PFC (nPFC): is a simple power supply (PS) with a diode rectifier and a smoothing capacitor that provides smooth voltage to a DC load. Examples of them are Compact Fluorescent Lamps (CFL) with rated power below $25 \mathrm{~W}$, and cell phone chargers. According to their current waveform shape, different current wave shapes are usually found in this category, as shown in fig 1a. Devices likes CFLs below 25W, Laptops and some LEDs have the blue current wave shape (nPFC 1), while battery chargers have usually the red wave shape (nPFC 2). Nevertheless, based on previous measurements much more wave shapes can be classified into nPFC category. The total harmonic emission of nPFC topology is usually very high (THDi>80\%) and the total power factor is usually below 0.6 (Table 1).

Passive PFC (pPFC): is a PS that has a combination of capacitors or inductors before or after the bridge diode rectifier in order to increase the power factor. They can be found e.g. in computer power supplies as well as in LED lamps. A typical waveform of pPFC computers is shown in fig. $1 \mathrm{~b}$.

Active PFC (aPFC): this topology is a PS with an advance circuit control that has the best performance related to power factor and harmonic emission. The resulting current wave is almost sinusoidal as shown in fig. 1c. This topology is used in modern energy-efficient appliances with higher power (e.g. computer power supplies, electric vehicle chargers). The waveform is also similar to PWM-based inverters as can be found e.g. in photovoltaic appliances.

Table 1. - Classification of the different Circuit Topologies [1].

\begin{tabular}{|c|c|c|}
\hline $\begin{array}{c}\text { Circuit } \\
\text { Topology }\end{array}$ & Power factor & THDi \\
\hline nPFC & Less than 0.6 & More than $80 \%$ \\
\hline pPFC & Between $0.7-0.9$ & $40 \% \leq$ THDi $\leq 80 \%$ \\
\hline aPFC & More than 0.9 & THDi $<40 \%$ \\
\hline
\end{tabular}

It is important to note that devices of the same topology do not have usually exactly the same current waveform due to small differences in the elements of the circuit or solutions implemented by the manufacturers [1]. This requires a certain robustness of the classification method.

\section{B. Current waveform distributions}

Fig 1d,-1f show the probability distributions of the current waveforms of different appliances. The analysis of the distributions is based on histograms of one waveform cycle settled in 20 bins. It can be seen that the current waveforms of the different appliances (fig 1a, fig. $1 \mathrm{~b}$ and fig. 1c) have different characteristics distributions (fig 1d, fig. 1e and fig. 1f ). They can be classified according to their behaviour with respect to the mean value, which is about zero for all devices due to the half-cycle symmetry.

Distribution of nPFC topology: Fig 1d shows the histogram of the nPFC 1 device presented in fig. 1a. The histogram of nPFC 2 is not shown, but very similar. The distribution has a high concentration of data around the mean value and little probability of occurrence of values far from it.

Distribution of pPFC topology: Fig 1e shows the histogram of the pPFC device presented in fig $1 \mathrm{~b}$. This distribution is similar to the distributions of nPFC devices but there are more values in amplitude different from the mean value, which results in a less relative concentration of values.

Distribution of aPFC topology: Fig if shows the histogram of the aPFC device presented in fig 1c. In this case, there is a completely different behaviour, and the data is less concentrated around the mean value. This is a kind of bimodal distribution and the mean value is in the middle of the two modes. The data concentration around the mean value is low. According to the waveform shape, this is the most similar to a sinusoidal waveform.

HOS is able to distinguish different features of distribution functions and consequently can help classifying the appliances and visualize them in the statistical space.

\section{HOS indices}

Different waveform shape exhibit different distributions and in consequence different statistical values (i.e. mean standard deviation, etc.). In this sense, the kurtosis and variance are measurements of the waveform shape and the skewness compute the deviation from the mean value.

Having a discrete series of values in the time domain (fig $1 \mathrm{a} / \mathrm{b} / \mathrm{c})$, statistics such as variance $(v)$, skewness $(s)$ and kurtosis $(k)$ can be computed cycle-by-cycle to characterize the waveform shape.

$$
\begin{gathered}
\text { Variance } \\
\text { Skewness } \quad s=\frac{\frac{\sum(x-\bar{x})^{2}}{n-1}}{\left[\frac{1}{n-1} \sum_{i=1}^{n}\left(x_{i}-\bar{x}\right)^{3}\right.} \\
\text { S } \left.\left.\sum_{i=1}^{n}-\bar{x}\right)^{2}\right]^{\frac{3}{2}}
\end{gathered}
$$




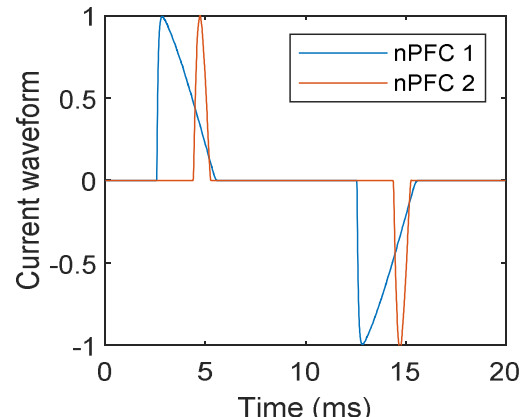

a) $\mathrm{nPFC}$ devices

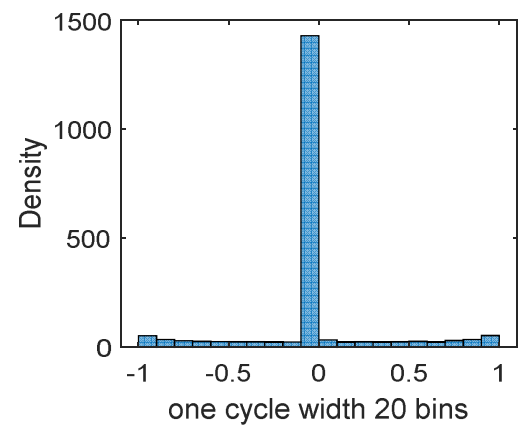

d) distribution of nPFC 1 device

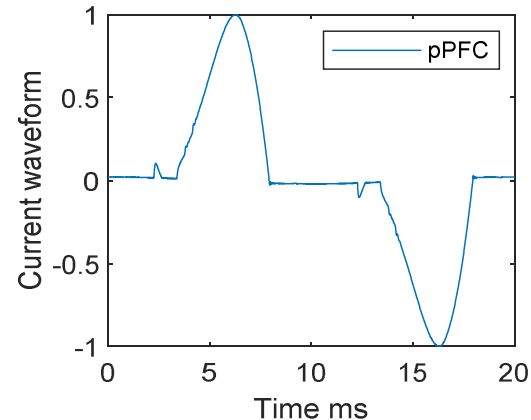

b) $\mathrm{pPFC}$ device

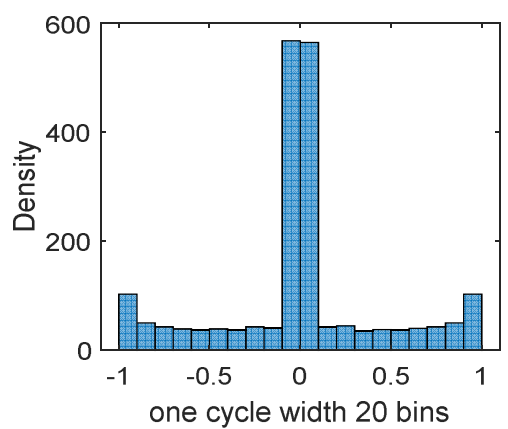

e) distribution pPFC device

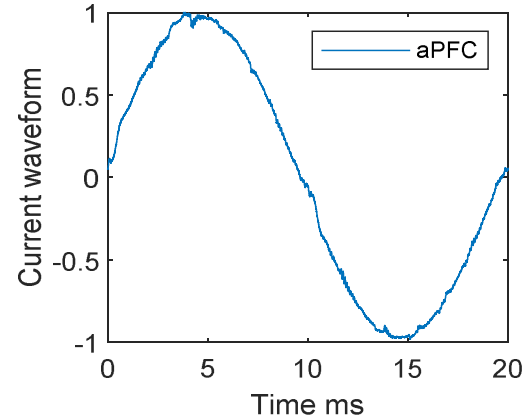

c) aPFC device

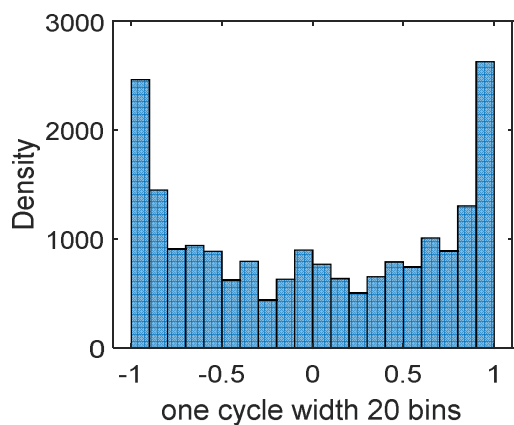

f) distribution aPFC device

Fig. 1. Normalized current waveforms and probability distributions of the normalized current waveforms of some appliances.

Kurtosis $\quad k=\frac{\frac{1}{N} \sum_{i=1}^{N}\left(x_{i}-\bar{x}\right)^{4}}{\left[\frac{1}{n-1} \sum_{i=1}^{n}\left(x_{i}-\bar{x}\right)^{2}\right]^{2}}$

where $\bar{x}$ is the sample mean, $\mathrm{n}$ the number of samples and $\mathrm{x}_{\mathrm{i}}$ the sample value $\mathrm{i}$.

It should be noted that variance and kurtosis are calculated based on a full power cycle, while skewness is calculated for a half-cycle only (skewness of one sinusoidal cycle is zero), because it has to be found more effective and robust monitoring results of the half cycle bias (some appliances just have a half-cycle) as result of the PFC. Table 2 gives the values of the selected HOS indices for each of the devices presented in fig. 1. In addition, the ideal values for a sinusoidal waveform are indicated as reference values [3], [5]. Skewness of a half cycle (sinusoidal waveform) tends to be negative.

Table 2. - Classification of the different circuit topologies of electronic appliances shown in fig 1 [1].

\begin{tabular}{|c|c|c|c|}
\hline \multirow{2}{*}{$\begin{array}{c}\text { Circuit } \\
\text { Topology }\end{array}$} & \multicolumn{3}{|c|}{ HOS classification } \\
\cline { 2 - 4 } & $\begin{array}{c}\boldsymbol{v} \\
(\mathbf{0 . 5})\end{array}$ & $\begin{array}{c}\boldsymbol{s} \\
\mathbf{( - \mathbf { 0 . 5 } )}\end{array}$ & $\begin{array}{c}\boldsymbol{k} \\
\mathbf{( 1 . 5 )}\end{array}$ \\
\hline nPFC 1 & 0.1 & 1.9 & 6.73 \\
\hline nPFC 2 & 0.04 & 3.09 & 13.52 \\
\hline pPFC & 0.2 & 0.92 & 3.54 \\
\hline aPFC & 0.36 & -0.09 & 1.93 \\
\hline
\end{tabular}

Appliances with nPFC have different current waveform (fig.1a). The first typical waveform (nPFC 1), has a sharkfin shape (triangular shape), the second waveform (nPFC 2 ) is more narrow and pointed. In both cases, variance is low, as most of the data is concentrated around the mean value. Kurtosis will clearly distinguish between the distribution tails with high positive values. The higher the kurtosis the steeper or pointed the distribution of the waveform (Table 2). In this sense, kurtosis of the second nPFC distribution is higher than the first one. Furthermore, the introduction of skewness can help to distinguish between the skewed left deviation of nPFC 1 (more asymmetric) and the distribution of nPFC 2.

Electronic devices with pPFC have right skewed distributions (mean is in the right) that can be identified by a lower skewness (fig.1b) compared with the nPFC appliances (fig.1a) that deviate more (wave shape and distribution) from a sinusoidal waveform. In addition, kurtosis and variance enable a classification by the mean value of one cycle. Variance is expected to be higher in nPFC equipment because the distribution is more disperse with respect to their mean value. However, in the same sense kurtosis of pPFC equipment must be lower in comparison with the nPFC devices. Devices that exhibit aPFC have the waveform shape close to sinusoidal shape. Therefore, aPFC devices show a higher variance and a lower kurtosis and skewness in comparison with the nPFC and pPFC devices

\section{Measurement and analysis framework}

The current waveform of 70 different electronic appliances with a variety of circuit topologies were obtained from PANDA equiPment hArmoNic Database (https://panda.et.tu-dresden.de) [7]. 


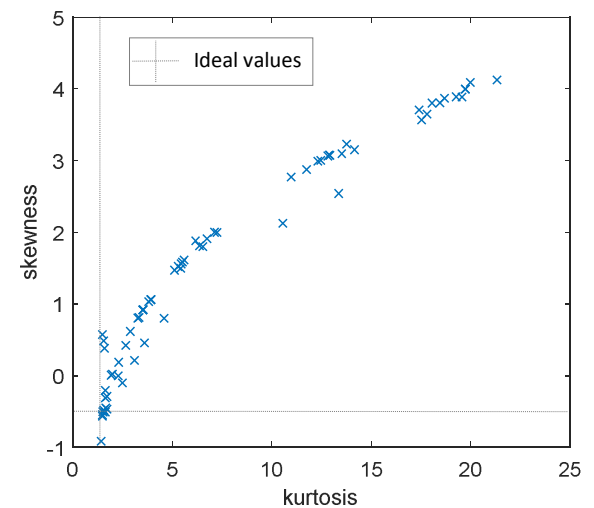

a) kurtosis vs skewness

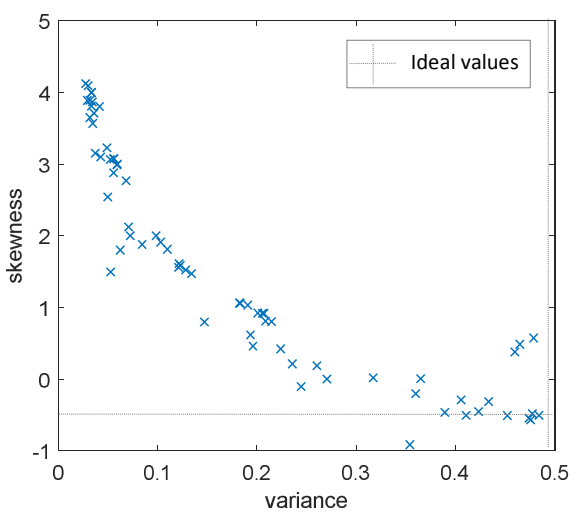

b) variance vs skewness

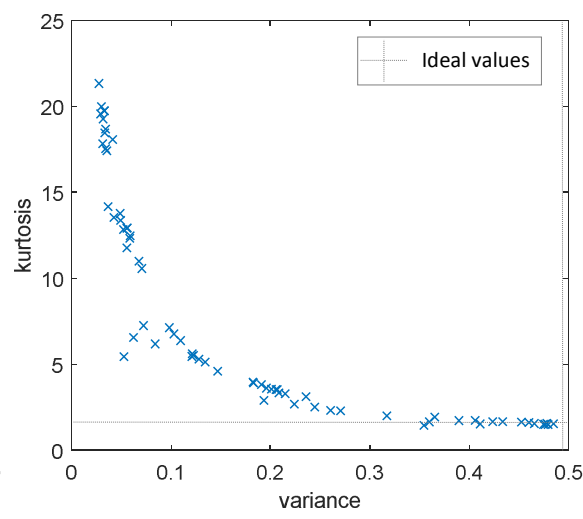

c) variance vs kurtosis

Fig. 2. Set of appliances with different circuit topology in HOS domain. Ideal values correspond to sinusoidal waveform.

All the selected devices were measured under normal operating conditions using a sinusoidal voltage waveform (THDu $<1.2 \%$ ). The signals have a sampling rate of $100 \mathrm{kS} / \mathrm{s}$.

The HOS indices presented in the previous section were calculated for each of the current waveforms using MATLAB. The method was implemented following the next steps:

1. Selection of the current waveform time series of each of the appliances from PANDA.

2. Normalization of the time series in order to scale the heterogeneous sets of data in the range [-1; 1]. The normalization facilitates the comparison of the different signals.

3. Computation of the individual indices. The variance and kurtosis are calculated for one cycle, while the skewness is calculated for the first half-cycle.

4. The statistics of steady-stated current waveforms are represented in different $2 \mathrm{D}$ graphs (in the HOS space), kurtosis vs skewness, variance vs skewness, variance vs kurtosis.

5. Different regions or clusters of different appliances are obtained in the HOS space.

In this sense, the kurtosis and variance are indices of the waveform shape. While more negative the skewness, it can be interpreted that circuit topologies are more near to aPFC distribution (sinusoidal waveform shape).

The proposed indices must yield intrinsic results of each of the appliances in the HOS space (fig. 2).

\section{Analysis results}

Fig. 2 presents 2D graphs for the different combinations: kurtosis vs skewness, variance vs skewness, variance vs kurtosis (see fig 2-a,b,c). Results from the HOS space show that some appliances exhibit a high kurtosis and skewness and small variance values while other appliances exhibit a low kurtosis and skewness and high variance value. According to the analysis of current waveshape, in the HOS space, the closer the current waveshape is to a sinusoidal waveform, the lower the kurtosis and skewness and the higher the variance.

Appliance identification in the HOS space were made a posteriori in order to understand the different circuit topology clusters and their behaviour in the different scenarios. Using the sinusoidal waveform as reference in the HOS space, the clusters presented in fig. 3 can be identified.

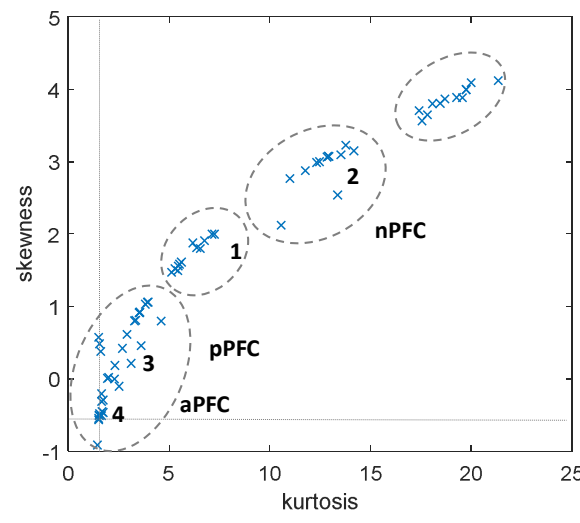

a) kurtosis vs skewness

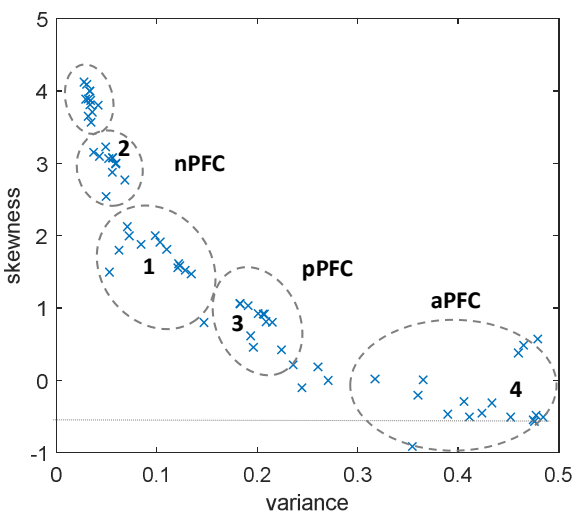

b) variance vs skewness

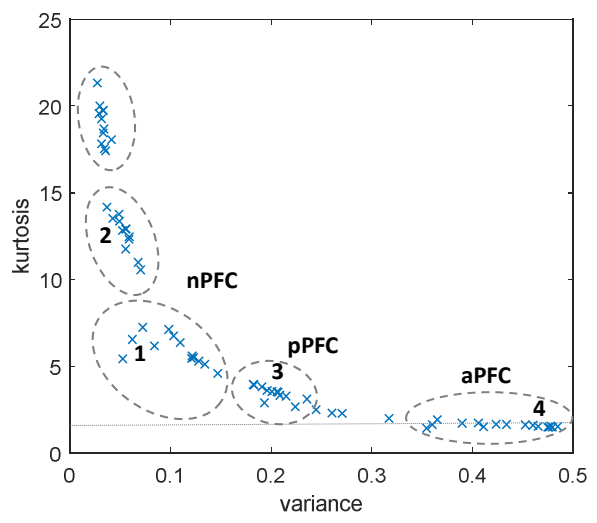

c) variance vs kurtosis

Fig. 3. Clustering of the circuit topologies according to the current prevailing waveforms in HOS domain. 
Clusters 1 and 2 with high kurtosis and skewness and low variance can be related to appliances with $\mathrm{nPFC}$ topologies. Cluster 4 with low kurtosis and skewness and a high variance can be linked to aPFC devices, as their distinctive distributions present a current waveform more close to the sinusoidal waveform. Cluster 3 is located between those described before and shows intermediate values of kurtosis, skewness and variance. In this sense, it contains appliances that present a relatively high concentration of data points around the mean, like it is typical for pPFC appliances.

Fig. 3 shows that kurtosis vs skewness, variance vs kurtosis and variance versus skewness can clearly distinguish different clusters and are consequently suitable to develop a classification method with different classification regions. This way HOS helps classifying the distribution features of appliances and visualize them through the statistic space.

Table 3 shows some more examples of appliances with different circuit topology and their corresponding values and cluster locations in fig 3 .

Table 3. - Classification of the different circuit topologies based on their cluster location [1].

\begin{tabular}{|c|c|c|c|c|c|c|}
\hline \multirow{2}{*}{ No } & \multirow{2}{*}{ Device } & \multicolumn{2}{|c|}{ HOS indices } & \multirow{2}{*}{$\begin{array}{c}\text { Circuit } \\
\text { Topology }\end{array}$} & Clusters \\
\cline { 3 - 5 } 1 & $\begin{array}{c}\text { CFL } \\
(16 \mathrm{~W})\end{array}$ & 0.7 & 1.8 & 6.7 & & $\begin{array}{c}100 \% \\
\mathrm{nPFC} 1\end{array}$ \\
\hline \multirow{2}{*}{2} & $\begin{array}{c}\text { LED } \\
(1.6 \mathrm{~W})\end{array}$ & 0.05 & 3.1 & 14.8 & $\mathrm{nPFC}$ & $\begin{array}{c}100 \% \\
\mathrm{nPFC} 2\end{array}$ \\
\hline \multirow{2}{*}{3} & $\begin{array}{c}\text { Desktop } \\
\text { Power }\end{array}$ & 0.2 & 0.8 & 4.5 & $\mathrm{pPFC}$ & $\begin{array}{c}80 \% \\
\mathrm{aPFC}\end{array}$ \\
& $\begin{array}{c}\text { Supply } \\
(400 \mathrm{~W})\end{array}$ & & & & & $\begin{array}{c}20 \% \\
\mathrm{pPFC}\end{array}$ \\
\hline \multirow{2}{*}{4} & $\begin{array}{c}\text { Electric } \\
\text { Vehicle } \\
(3600 \mathrm{~W})\end{array}$ & 0.49 & -0.5 & 1.5 & $\mathrm{aPFC}$ & $\begin{array}{c}100 \% \\
\mathrm{aPFC}\end{array}$ \\
\hline
\end{tabular}

In addition, based on fig. 3 typical ranges can be derived, in which devices with common topologies can be expected. E.g. for devices with aPFC variance is usually in the range $v=0.4$.. 0.5 , skewness in the range $\mathrm{s}=-1 . .1$ and kurtosis around $\mathrm{k}=1.5$. This area is close to the ideal values of sinusoidal waveform $(\mathrm{v}=0.5, \mathrm{~s}=-0.5, \mathrm{k}=1.5)$, also indicated in fig. 3. Consequently, they are the most linear devices.

It should be noted that due to the high diversity in circuit designs and consequently also the resulting current waveforms, a $100 \%$ reliable classification of circuit topologies is not possible. E.g. the nPFC region exhibits different clusters that would be indicative of different waveform shapes within that circuit topology as results of solutions implemented by the manufacturers. The HOS indices and the introduced classification can serve as a reliable indicator for the level of nonlinearity of a device and consequently its severity to the network. Moreover the HOS indices are efficient features to assess the current waveshape of a device with relation to the ideal sinusoidal one. E.g. left and right skewed waveforms can be distinguished in the HOS space helping to distinguish between nPFC 1 (high skewness) and pPFC (lower skewness). The layout of the method is illustrated in Fig. 4 , where all the stages are summarized in the process flow diagram, the feature extraction and the classification within the 2D and 3D HOS graphs. A classification in the 3D space is deployed in Fig. 5.

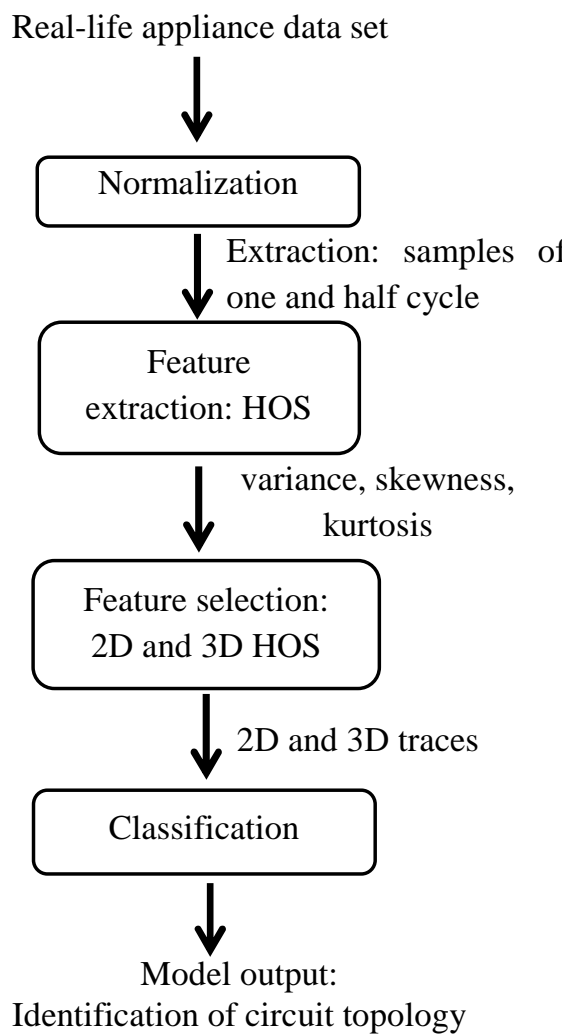

Fig. 4. Proposed method for circuit topology classification based on HOS.

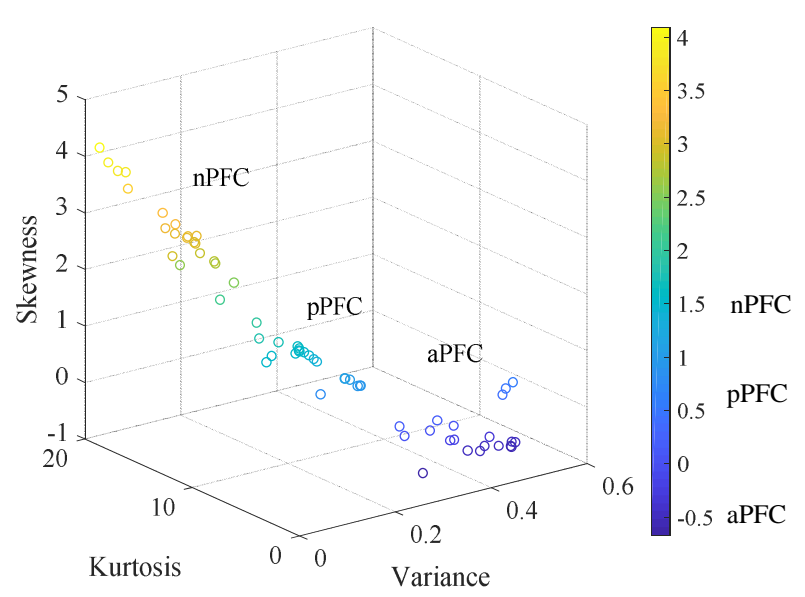

Fig. 5. Classification of the circuit topologies in the HOS domain for different appliances. The color bar represents the scale for the skewness. 
A classification analysis of the devices taking all three HOS indicators into account could improve the classification as it can be derived from the 3D plot in Fig. 5. This must be a motivation for further studies in circuit topology classification based on HOS.

From the computational point of view, it can be easier to calculate all the indices and estimate them from a halfcycle.

\section{Conclusions}

This paper applies HOS in order to classify the current waveshape of individual appliances, which has been measured under sinusoidal supply voltage at nominal magnitude and normal operating conditions as it is required in IEC 61000-3-2 for compliance assessment.

HOS classification has proven to be a useful tool, because the indices are calculated in the time domain without the need of complex operations like Fourier transform. In addition, statistics help comprise information on the original waveform shape and their probability density distribution function.

In addition, HOS help detect different commonly used circuit topologies without PFC, with passive PFC and with active PFC. Respective clusters have been initially identified in this paper, but determining cluster limits for the different topologies must be further studied.

The proposed method is able to identify the level of nonlinearity based on the measured electrical current, which can be associated with a single device, but also a mix of multiple devices, a whole customer (e.g. a household) or even network.

As the method requires only sampled waveform data in time domain it could be easily implemented e.g. in smart meters, which could improve the measurement tools for advance metering infrastructure (AMI) applications not requiring additional computational performance.

The automatization of the classification method based on application of machine learning and/or pattern recognition techniques can improve the usefulness significantly. It can be used to identify "exotic" devices that do not follow common current waveforms and distributions. Moreover the automatic identification of non-typical customer behaviour can be a helpful tool for network operators in the future. In addition, the method could be used in a nonintrusive load monitoring system (NILMS) or to detect different operating states of devices (e.g. washing machines).

Next steps will include the analysis of the robustness of the HOS indices regarding supply voltage conditions (field application) as well as its application to a mix of devices in a frame of hybrid waveforms.
We would like to express our acknowledgement to the Spanish Ministry of Economy, Industry and Competitiveness and the EU (AEI/FEDER/UE) via the funding of the project TEC2016-77632-C3-3-R. In addition, our gratitude goes to the Andalusian Government for supporting the Research Group PAIDITIC-168, in Computational Instrumentation and Industrial Electronics (ICEI). The University of Cadiz for financing the research internship through their program to support research staff in research centres of recognized prestige.

\section{References}

[1] A. M. Blanco, M. Gupta, A. Gil De Castro, S. Rönnberg, J. Meyer; Impact of flat-top voltage waveform distortion on harmonic current emission and summation of electronic household appliances, International Conference on Renewable Energies and Power Quality (ICREPQ18), 2018.

[2] J. Meyer, A. M. Blanco, Max Domagk, Peter Schegner; Assessment of Prevailing Harmonic Current Emission in Public Low-Voltage Networks, IEEE Transaction on Power Delivery, vol. 32, $\mathrm{n}^{\circ}$ 2, 2017.

[3] O. Florencias-Oliveros, J.J. González-de-la-Rosa, A. Agüera-Pérez, J. C. Palomares-Salas; Power quality event dynamics characterization via $2 \mathrm{D}$ trajectories using deviations of higher-order statistics Measurement, vol. 125, p. 350-359, 2018.

[4] M.V. Ribeiro, C. A. G. Marques, C. A. Duque, A. S. Cerqueira, J. L. R. Pereira; Detection of disturbances in voltage signals for power quality analysis using HOS; EURASIP J. Adv. Signal Process., 2007.

[5] A. Agüera-Pérez, J. C. Palomares-Salas, J.J. González-de-la-Rosa, J. M. Sierra-Fernández, D. Ayora-Sedeño, A. Moreno-Muñoz; Characterization of electrical sags and swells using higher-order statistical estimators; Measurement, $\mathrm{n}^{\circ} 44$, pp. 14531460, 2011.

[6] Juan D.S. Guedes, Danton D.Ferreira, Bruno H.G. Barbosa; A non-intrusive approach to classify electrical appliances based on higher-order statistics and genetic algorithm: a smart grid perspective, Electric Power Systems Research, vol. 140, 2016.

[7] A. M. Blanco, E. Glasch, J. Meyer, P. Schegner; Webbased platform for exchanging harmonics emission measurements of electronic equipment; IEEE 15th Internacional Conference on Harmonics and Quality of Power-ICHQP, 2012.

\section{Acknowledgement}

